\title{
Generating and Maintaining Activity-based Cost Estimates with Feature-Based Product Models
}

\author{
by
}

\author{
Sheryl Staub-French ${ }^{1}$ and Martin Fischer ${ }^{2}$
}

\begin{abstract}
Understanding how the building design influences construction costs is a challenging task for estimators. Estimators must recognize the design conditions that affect construction costs and customize the cost estimate accordingly. Estimators have different preferences for how and when to adjust a project's activities, resources, and resource productivity rates that form the basis of a cost estimate. Current tools and methodologies lack ways to help estimators customize construction cost information according to their preferences and maintain cost estimates as the design changes based on those preferences. This paper describes the activity-based cost estimating process we formalized to help estimators customize a project's activities, resources, and resource productivity rates based on their preferences and the particular features in a given product model. We implemented and tested the process in a prototype called Activity-based Cost Estimating (ACE). ACE creates a set of projectspecific activities that know why they are needed in the cost estimate, what feature requires their execution, what resources are executing the activity and why, and what their labor and material costs are. Our tests show that ACE helps estimators to generate and maintain cost estimates more completely, consistently, and quickly than state-of-the-art cost estimating software.
\end{abstract}

\section{KEYWORDS: Cost Estimating; Information Technology; Construction; Product Features}

\section{INTRODUCTION}

It is the cost estimator's task to determine how a building design influences construction costs. Estimators must determine what design conditions are important (i.e., incur a cost), when they are important, and how they affect construction costs when creating cost estimates. Construction cost estimates are used to assist designers, owners, and builders of facilities in resolving a variety of decisions, such as evaluating the cost of different design alternatives, budgeting construction costs, and establishing the cost impact of design changes. Consequently, it is critical that cost estimators provide detailed and accurate cost estimates in a timely manner to support project teams in making these different decisions.

Estimators using state-of-the-art estimating software can establish a relationship between a component in a product model and a cost item in a cost-estimating database when creating a cost estimate (Timberline 2001). These relationships help estimators to take off quantities automatically by representing the component properties that affect construction costs. However, this representation is incomplete because it does not represent the estimator's rationale for how the component properties affect specific cost information, and it does not represent other design conditions and their impact on a component's cost. Consequently, generating and maintaining cost estimates today is a largely manual, errorprone, and time-consuming process.

This paper describes the formalization of an activity-based cost estimating process that leverages the rich representation of standard product models to help estimators generate and maintain construction cost estimates. This research is based on a completed research project and was motivated by our experiences with state-of-the-art integrated estimating software on real projects. This work addresses

\footnotetext{
${ }^{1}$ Assistant Professor, Department of Civil Engineering, University of British Columbia, 2324 Main Mall, Vancouver, BC, Canada, V6T 1Z4. Voice: 604-827-5118, Fax: 604-822-6901, sherylsf@civil.ubc.ca.

${ }^{2}$ Associate Professor, Department of Civil and Environmental Engineering and (by Courtesy) Computer Science, Director, Center for Integrated Facility Engineering, Terman Engineering Center, Room 292, Stanford University, Stanford, CA 94305-4020. Voice: 650-725-4649, Fax: 650-725-6014, fischer@stanford.edu.
} 
conference themes related to computer integrated building processes and construction process modeling and simulation.

\section{CASE STUDY}

This section describes a case study to illustrate the requirements for automated support of the cost estimating process. The case study is based on a drywall estimator's process for estimating the labor costs for one of the rooms in an office project shown in Figure 1. Figure 2 shows an estimator's rationale for adjusting the activities, resources, and resource productivity rates to account for the cost impacts of specific design conditions.

The case study demonstrates that there are different types of design conditions that affect construction costs. Design conditions can be based on properties of components (e.g., the 'curvature' of the wall), intersections of components (e.g., the 'structural penetration' resulting from the intersection of the wall and beam), and groupings of components (e.g., the 'grouping of walls' based on component similarity). The case study also demonstrates that design conditions can affect construction costs in different ways. Design conditions can affect the requirement for activities (e.g., the 'structural penetration' requires the activity Apply Caulk), design conditions can affect when a resource is appropriate in an activity (e.g., the height of the wall affects the need for Rolling Scaffolding), and design conditions can affect a resource's ability to execute an activity effectively (e.g., the similarity of the walls leads to an increase in crew productivity).

Estimators using state-of-the-art cost estimating software cannot represent many of the design conditions that affect construction costs (e.g., structural penetrations and component similarity). Furthermore, estimators using state-of-the-art cost estimating software cannot explicitly represent the specific cost information affected and the way it is affected by different design conditions (e.g., resource use or execution). Consequently, estimators have to manually identify most design conditions and manually adjust the project's activities and resources accordingly. For a large project, it is typically too time-consuming to make these project- specific adjustments manually for all the different design conditions in a given product model. Consequently, estimators often employ ad hoc methods (e.g., adjusting the crew productivity rates of all the "Install Metal Studs" activities to account for one wall's curvature) and overlook the cost impact of different design conditions (e.g., overlook cost impacts resulting from openings). Moreover, estimators often do not have the time to provide specific feedback to designers on the cost implications of their design decisions (e.g., the cost implications of a specific wall height). The lack of a formal process and automated support leads to inconsistencies and inefficiencies in the cost estimating process and resulting cost estimate, and limits the ability of estimators to help designers develop cost-effective designs.

\section{GENERATING AND MAINTAINING COST ESTIMATES USING ACE}

The activity-based cost estimating process we formalized helps estimators to generate and maintain cost estimates quickly and consistently based on the design conditions in a given product model. We implemented and tested this process in a prototype called Activity-based Cost Estimating (ACE). The main challenges associated with providing automated support of the cost estimating process are that different design conditions exist in any given product model, that different design conditions affect construction costs in different ways, and that estimators have different preferences for how and when to adjust construction costs to account for different design conditions.

We developed the activity-based cost estimating process by abstracting the design conditions estimators consider and the different ways estimators adjust activities and resources to account for different design conditions. We use features to describe the specific part of the design that estimators care about, design conditions to describe when features are important to estimators, and activities to describe how features impact production and hence, construction cost. By leveraging the activity-based cost estimating process, ACE allows estimators to represent their rationale for relating features of a building product model with construction 
activities to enable automated support of the cost estimating process.

Figure 3 shows the three different modules of the activity-based cost estimating process:

(1) Instantiate Features: Identify the relevant cost-driving features in the input product model and instantiate the features to create an estimator-focused feature-based product model.

(2) Customize Activities and Resources: Customize the activities and resources for each component being estimated based on the estimator's rationale and the particular features in the estimator-focused feature-based product model.

(3) Generate and Maintain Construction Costs: Calculate each activity's quantities and duration to determine the activity's cost. If the estimate is based on a revised design, identify the cost information affected and reconcile the activities and resources so that the design and estimate remain in balance. The output of this process is a set of resource-loaded and costloaded activities that are explicitly related to the features in the input product model.

\section{M1: Instantiate Features}

The motivating case shows that different types of design conditions affect construction costs. Estimators consider properties of components, intersections of components, and groupings of components when creating cost estimates. The purpose of the first module is to transform designer-focused product models into featurebased product models that support cost estimating. The input product model is represented using the industry standard Industry Foundation Classes (IFC) (IAI 2001). $\mathrm{ACE}$ identifies the cost-driving features in the input IFC-based product model to create an estimator-focused feature-based product model.

The IFC's explicitly represent components, attributes of components, and relationships between components in building product models (IAI 2001). However, they do not explicitly represent many of the design conditions that affect construction costs, such as penetrations and component similarity. We use features to represent the design conditions that are important to cost estimators of building construction. Product features are used extensively in manufacturing to describe the geometric forms or entities in a product model that are important in some aspect of the manufacturing process (Cunningham and Dixon 1988). However, the feature representations developed in the manufacturing industry do not fully support the representation of building product models. Specifically, building product models contain different features and different types of products. Our research applies the manufacturing concept of features to building construction and extends it to represent the features that are useful to cost estimators.

We modeled three different types of features in this research: (1) component features (e.g., 'walls'), (2) intersection features (e.g., 'structural penetrations'), and (3) macro features (e.g., 'groupings of components based on similarity'). We formalized a feature ontology that represents the different attributes of each feature type and enables estimators to represent feature instances according to their preferences (Staub-French 2002). The feature ontology provides the map to relate an IFCbased product model to an estimator-focused product model. We represent features in a project-independent way so that they can be reused from project to project to identify the relevant features given an IFC-based product model. -

ACE leverages the feature ontology to provide a framework for estimators to represent their preferences for naming features, specifying relevant component intersections, defining component similarity, and specifying the features that affect a specific component's construction costs. For example, the estimator from the motivating case can represent the "structural penetration" as a feature that results from the intersection of 'walls' and 'beams,' and specify that this feature is important for constructing 'walls.' ACE analyzes the geometry and topological relationships between the components in the input IFCbased product model to identify the costdriving features specified by the estimator. Hence, ACE enriches current standard building product models by representing the features of building product models that affect construction costs. 


\section{M2: Customize Activities and Resources}

The case study shows that estimators adjust the project's activities, resources, and resource productivity rates to account for the cost impact of different features. The purpose of the second module is to help estimators customize the activities and resources in a cost estimate according to their preferences based on the specific features in a given product model.

Prior research efforts demonstrate that cost estimates can be generated directly from 3D models (Laitinen 1998; Aouad et al. 1994; Aouad et al. 1997, Staub-French and Fischer 2001). However, these research efforts do not customize construction cost information for specific design conditions in a given product model, and they do not account for different estimator preferences. Other research efforts recognize design conditions that affect construction costs and customize the activity's resources and resource productivity rates accordingly (Fischer 1991; Thomas and Zavrski 2000; Hanna et al. 1992). However, these research efforts do not represent and account for different estimator preferences when customizing the project's resources and resource productivity rates to the design conditions in a particular product model.

We abstracted the common attributes of estimators' rationale for how and when different design conditions affect construction costs and developed templates to capture this estimating knowledge from estimators (StaubFrench 2002). The templates allow estimators to specify the features that affect activities (Activity Specification templates) and the features that affect resources (Resource Specification templates). The templates provide a structured way for estimators to represent the specific impact different features have on a project's activities and resources. Estimators input their rationale once in the Activity and Resource Specification templates and ACE reuses this knowledge from project to project when generating and maintaining cost estimates.

In ACE, we implemented a formal process that automatically customizes activities and resources when generating and maintaining cost estimates for estimator-focused feature- based product models (Staub-French 2002). For each feature in the input product model, ACE identifies the relevant Activity Specifications and adds the specified activity to the estimate. Then, ACE assigns resources to the activities and adjusts the resources' productivity rates according to the estimator's preferences in Resource Specifications and based on the specific features in the featurebased product model. The output of the second module is a set of project-specific resource-loaded activities that are explicitly related to the estimator-focused feature-based product model and the estimator's rationale.

\section{M3: Generate and Maintain Construction Costs}

The purpose of the third module is to generate and maintain construction costs given the input resource-loaded activities and related features. Calculating the construction costs for resourceloaded activities is a straightforward process. However, the explicit relationships between features, activities, resources, costs, and the estimator's rationale in Activity and Resource Specifications enable the maintenance of cost estimates if the design changes.

Many research efforts have developed computer tools that automatically generate the project-specific relationships between components, activities, resources, and costs (Laitinen 1998; Aouad et al. 1994; Aouad et al. 1997; Froese 1992). However, they do not represent why components, activities, resources, and costs are related and when the relationships are needed.

Our research extends existing formalisms of construction processes that define activities as objects $<\mathrm{O}>$, actions $\langle\mathrm{A}\rangle$, and resources $<\mathrm{R}>$ (Darwiche et al. 1988; Aalami 1998). Our research extends this formalism by generating activities that also know what feature $<\mathrm{F}>$ requires the activity's execution and how much the activity costs $<\mathrm{C}>$ to create an integrated $<$ FOARC $>$ model consisting of activities that explicitly relate features, objects, actions, resources, and costs.

Each activity generated by ACE knows what feature requires its execution, the material and resource cost implications of the activity, the estimator's rationale for adding the activity, 
what resources are executing the activity and why, and how particular features affect the resources' productivity rate. Consequently, ACE can help estimators to identify the cost information affected by design changes and calculate the corresponding cost impact of design changes. Our tests show that the cost estimating process implemented in ACE and the resulting integrated $<$ FOARC $>$ model enables estimators to generate and maintain construction cost estimates from feature-based product models more completely (i.e., less ad hoc and with fewer omissions), consistently, and quickly (Staub-French 2002).

\section{CONCLUSIONS}

This paper described an activity-based cost estimating process that creates an integrated model consisting of activities that explicitly relate features, objects, actions, resources, and costs, and the estimator's rationale for relating this information. This process helps estimators to generate and maintain construction cost estimates and avoid ad hoc and error-prone methods that lead to inconsistencies and inefficiencies in the cost estimating process.

The formalisms developed and implemented in ACE take an essential step toward creating software tools that can help project teams to maintain integrated models of a project's scope, schedule, and cost. Understanding the relationships between this information is critical to managing the design and construction process. Future research directions should address other types of features and factors exogenous to product design, such as site characteristics and resource skill and availability.

\section{REFERENCES}

Aalami, F. (1998). "Using Method Models to Generate 4D Production Models," PhD Thesis, Stanford University, Stanford.

Aouad, G., Betts, M., Brandon, P., Brown, F., Child, T., Cooper, G., Ford, S., Kirkham, J., Oxma, R., Sarshar, M., and Young, B. (1994). "ICON: Integration of Construction Information." Department of Surveying and Information Technology Institute, University of Salford, Salford.
Aouad, G., Child, T., Marir, F., and Brandon, P. (1997). "Open Systems for Construction (OSCON), draft industry report." Department of Surveying, University of Salford, Salford.

Cunningham, J.J. and Dixon, J.R. (1988). "Designing with Features: The Origin of Features," ASME Computers in Engineering Conference, San Francisco, CA, Aug. 1988, 237-243.

Darwiche, A., Levitt, R., and Hayes-Roth, B. (1989). "OARPLAN: Generating Project Plans by Reasoning about Objects, Actions and Resources.” AI EDAM, 2(3), 169-181.

Fischer, M. (1991). "Constructibility Input to Preliminary Design of Reinforced Concrete Structures." Technical Report 64, Center for Integrated Facility Engineering, Stanford.

Froese, T. M. (1992). "Integrated ComputerAided Project Management Through Standard Object-Oriented Models." PhD Thesis, Stanford University, Stanford.

Hanna, A. S., Willenbrock, J.H., and Sanvido, V. E. (1992). "Knowledge Acquisition and Development for Formwork Selection System." Journal of Construction Engineering and Management, ASCE, 118(1), 179-198.

International Alliance of Interoperability (IAI) (2001). "IFC 2x Extension Modeling Guide", Available from http://www.iai.org.uk.

Laitinen, J. (1998). "Model Based Construction Process Management." $\mathrm{PhD}$ Thesis, Royal Institute of Technology. Stockholm, Sweden.

Staub-French, S., and Fischer, M. (2001). "Industrial Case Study of Electronic Design, Cost, and Schedule Integration." Technical Report 122, Center for Integrated Facility Engineering, Stanford.

Staub-French, Sheryl (2002) "Feature-Driven Activity-based Cost Estimating." PhD Thesis, Stanford University, Stanford.

Thomas, H.R. and Zavrski, I. (1999). "Construction Baseline Productivity: Theory and Practice." Journal of Construction 
Engineering and Management, American Society of Civil Engineers, 125(5), 295-303.

Timberline Software Company (2001). Precision Estimating Extended and CAD Integrator, Users Documentation, Beaverton, Oregon.

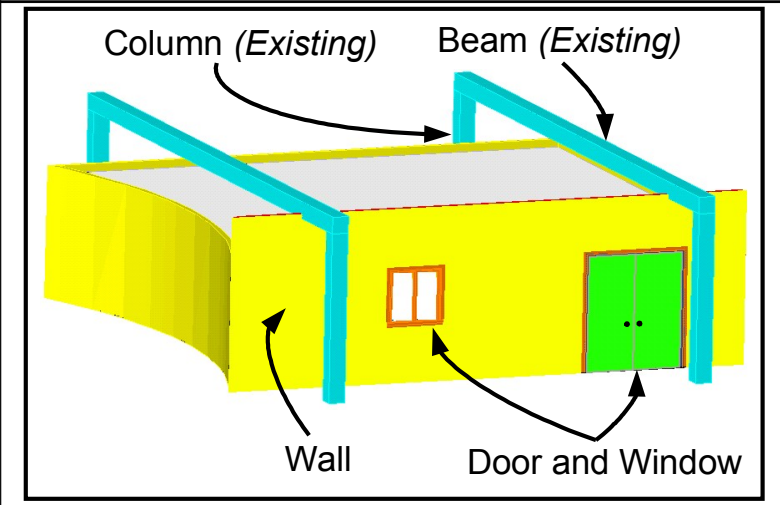

Figure 1. Building components in the office project case study.

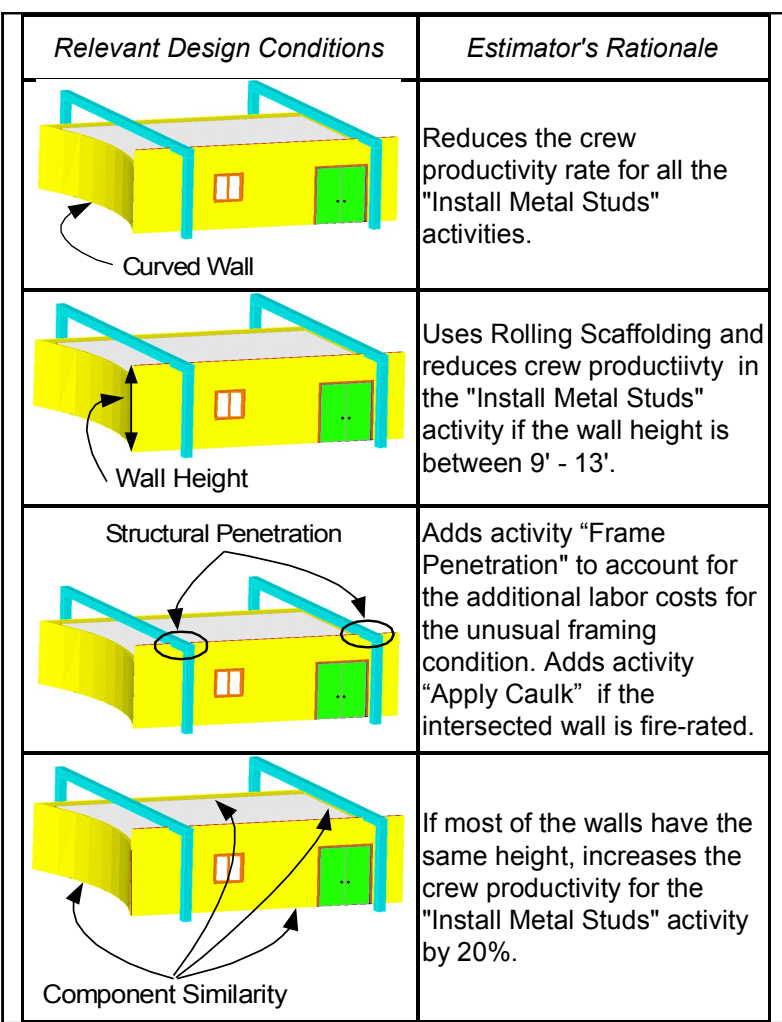

Figure 2. Estimators' rationale for adjusting the activities, resources, and resources' productivity rates to reflect the cost impact of specific design conditions in the cost estimate.

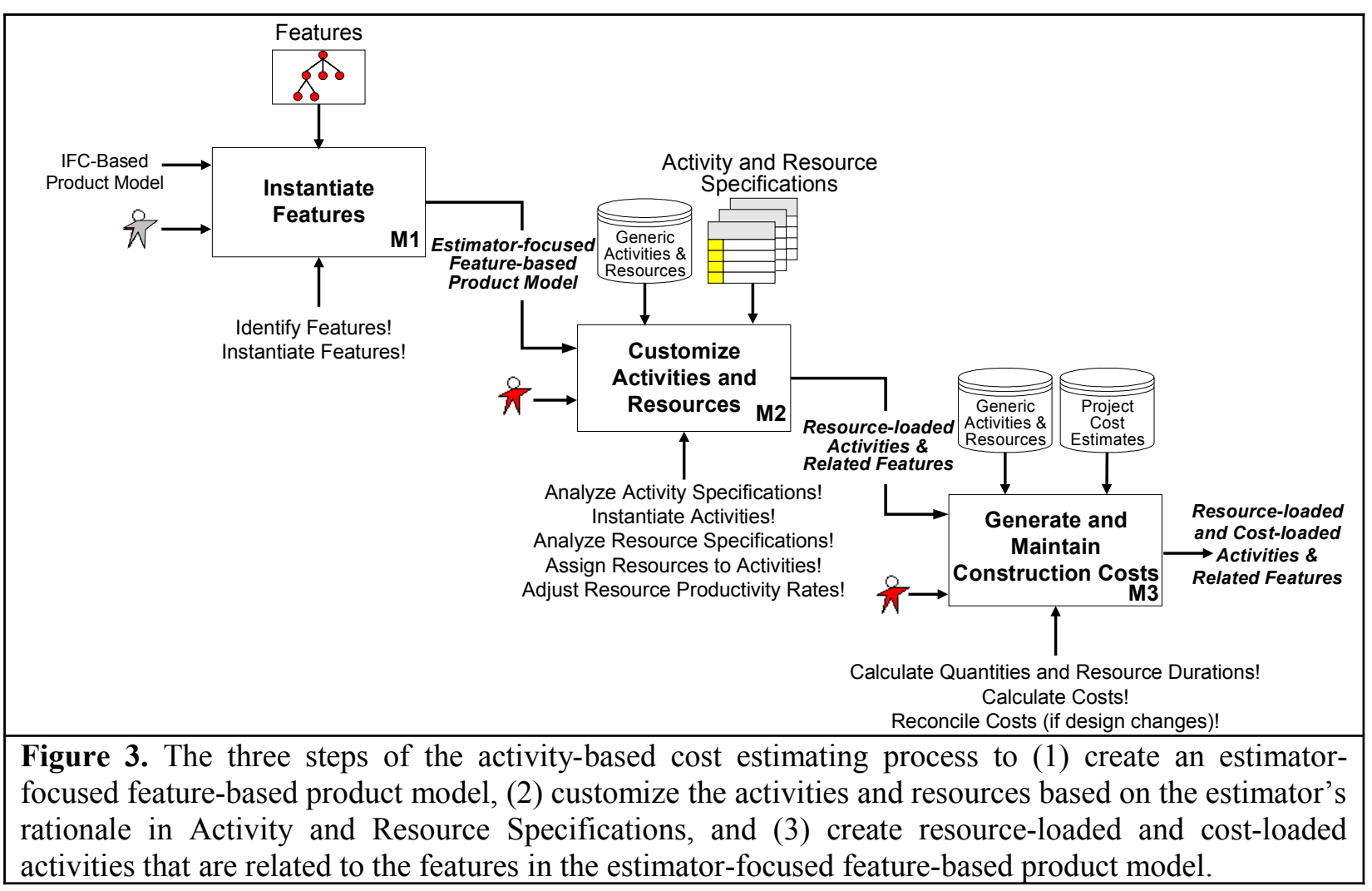

